torical survey, generic diagnosis and synonomy and culture technique. The historical survey traces the evolution of the taxonomy of the genus from 1792 to the present day. In the generic diagnosis section the question of penicillate genera which have previously been included in the genus, or penicillia which have been erroneously referred to other genera, is discussed, and the concept of the genus has been more clearly defined. The culture technique section deals in detail with the observations to be made and the cultural conditions under which they should be made, in order adequately to describe the essential characteristics of colonies from a diagnostic point of view. A chapter on cultivation of penicillia and a short account of penicillin completes Part I.

The second part which occupies approximately 600 pages gives an exhaustive treatment of the sections, series and species into which the genus has been divided. The use of the manual is explained, and the bases of the classification expressed in a key leading to the identification of the four ' sections,' Monoverticillata, Asymmetrica, Biverticillata-Symmetrica and Polyverticillata and the 'series' into which these are respectively subdivided. This is followed by detailed treatments of each section. The diagnostic keys and detailed descriptions in this part are designed to facilitate accurate identification down to species even by those who are relatively inexperienced in the genus.

The third part of roo pages includes an account of the genera Gliocladium, Paecilomyces and Scopulariopsis which, though removed from the genus Penicillum, are treated in the same comprehensive manner. There is an excellent topical bibliography, a general bibliography of some 900 references, and lists of accepted and rejected species. There is a very adequate index with the more significant pages for any subject indicated by heavy type.

An important feature of the book is the wealth of illustrations. There are ten coloured plates, a relatively enormous number of photographs of plate cultures, organisms growing on their hosts and large scale detail. The numerous excellent line drawings of habit and details of the penicillin in the different sections are very valuable aids to determination. The paper is good and the volume is attractively bound.

It would perhaps be difficult to over-estimate the importance of the manual. It is the only authoritative work on the subject and one of the most outstanding contributions of modern times to the science of Mycology.

$$
\text { W.H.W. }
$$

\section{THE RADCLIFFE INFIRMARY HANDBOOK}

Pp. I 50. Oxford: University Press. 1949. 7s. 6d.

The foreword by Professor A. D. Gardner, Regius Professor of Medicine, tells us that the first edition, edited by Drs. Robb-Smith and Cook, appeared in typescript only in 1940. This 1949 edition, prepared by Drs. Sidney Truelove and
Cook, is, therefore, the first in print. It is primarily intended for the use and information of house officers of the Infirmary during their relatively short periods of office, but it contains much likely to be helpful to anyone associated with hospital organization.

There are interesting notes on the foundation of the Infirmary; its development is sketched and the staff is listed. The duties of junior medical officers are set out with details of the byelaws affecting them and the procedures to be followed in any set of circumstances from admissions to outbreaks of infection. There is a timetable of outpatient clinics and official ward rounds and an excellent chapter on note-taking. Details of the facilities available in the clinical, pathological and biochemical laboratories and X-ray and anaesthetics departments follow and the actions necessary to obtain their services are outlined clearly. The closing chapters deal with the dispensary, sterile products, medical photography and how to obtain electrocardiograms.

The book altogether is worthy of the institution whose name it bears and the pattern in a proportionately less ambitious form could well be followed in many other hospitals.

$$
\text { J.C.F. }
$$

\section{ADVANCES IN PEDIATRICS (Vol. 4)}

Edited by S. Z. Levine. Pp. ix +316 , illustrated London and New York: Interscience Publishers I949. 52s.

The editors of Advances in Pediatrics, Volume 4 -successfully maintain the high standard set in the previous volumes by again providing a series of monographs, seven in all, on topics of importance to pediatricians.

S. Siwe's (University of Lund) critical review of the reticulo-endothelioses of children warns against too rigid a classification at a time when it is wiser to collect and note clinico-pathological features, and the similarity connecting different types, Gaucher's, Niemann-Pick's, Hand-Schüller-Christian's diseases, eosinophilic granuloma, and Letterer-Siwe's disease. His remarks on non-lipid reticulo-endotheliosis (Letterer-Siwe's disease) are specially valuable, as the condition was first described as recently as r 933 . The non-lipid reticuloses, he states, are invariably general reactions with enlargement of the spleen, liver, lymph nodes, fever and cutaneous haemorrhages as early signs, and a secondary anaemia, in children of a few months to three years old, and with a course of a few weeks to several months, terminating fatally.

A full and clear exposition of that rare condition, cystinosis, or Lignac's disease, is provided by $\mathbf{E}$. Freudenberg (Basle). This inborn error of metabolism is a disturbance in the breakdown of a number of the sulphur-containing amino acids. The infantile form commences at the end of the first, or beginning of the second year, with signs of thirst, polyuria, vomiting, arrested growth and intractable rickets. Cystinuria is inconstant, but signs differentiating the condition in vivo from 